\title{
Um estudo do suporte quantitativo necessário para a operacionalização da MCC
}

\author{
Angélica Alebrant Mendes ${ }^{\mathrm{a} *}$, José Luis Duarte Ribeiro ${ }^{\mathrm{b}}$ \\ a*angelica@producao.ufrgs.br, UFRGS, Brasil \\ bribeiro@producao.ufrgs.br, UFRGS, Brasil
}

\begin{abstract}
Resumo
Este artigo investiga o suporte quantitativo necessário à operacionalização da manutenção centrada em confiabilidade (MCC). Inicialmente, foram identificadas na literatura dez atividades consideradas essenciais para a operacionalização da MCC e os métodos quantitativos que podem dar suporte a essa metodologia. A seguir, foram realizadas entrevistas em empresas manufatureiras a fim de verificar a utilização dos métodos descritos na literatura e identificar outros métodos usados. Como resultado, foi elaborado um quadro que associa as atividades essenciais da MCC aos métodos quantitativos, juntamente com uma breve descrição das contribuições de cada método. Entre os métodos quantitativos identificados, destaque para o uso de distribuições de probabilidade, que suporta várias atividades da MCC. Os métodos de engenharia econômica e a simulação de Monte Carlo também merecem destaque, pois permitem análises mais sofisticadas associadas a custo e desempenho de sistemas produtivos sujeitos à manutenção.
\end{abstract}

Palavras-chave

Manutenção centrada em confiabilidade. Métodos quantitativos. Indústria metal-mecânica. Indústria eletrônica.

\section{Introdução}

A competitividade entre as empresas tem levado as organizações a buscar novas estratégias de mercado e reduzir seus custos operacionais. A melhoria das atividades de manutenção pode não somente reduzir esses custos, mas proporcionar melhorias na segurança, nas relações com o meio ambiente e no atendimento aos requisitos normativos da empresa (BLO0M, 2006; RAUSAND, 1998).

Tradicionalmente, a manutenção é planejada utilizando-se a experiência dos funcionários envolvidos e a orientação contida nos manuais dos fabricantes dos equipamentos (LAFRAIA, 2001; RAUSAND, 1998). Sabe-se que grande parte dos funcionários acredita na premissa de que as falhas ocorrem devido ao desgaste no tempo e que revisões ou substituições periódicas reconstituem a condição inicial do equipamento (WILMETH; USREY, 2000). Contudo, estudos feitos na aviação determinaram que apenas $11 \%$ dos componentes falham devido a desgaste e fadiga (LAFRAIA, 2001; WILMETH; USREY, 2000). Paralelamente, no que concerne às orientações contidas nos manuais dos fabricantes, corre-se o risco de essas recomendações não serem baseadas em dados reais, pois alguns fabricantes de equipamentos, a fim de maximizar vendas de componentes ou minimizar sua responsabilidade, orientam intervalos curtos de revisão e substituição (LAFRAIA, 2001; RAUSAND, 1998).

A manutenção centrada em confiabilidade (MCC) é um programa que combina técnicas de engenharia em uma abordagem sistemática a fim de garantir que os equipamentos fabris mantenham suas funções originais (FOGLIATTO; RIBEIRO, 2008). Para Hansen (2006), a MCC desenvolve estratégias de manutenção que combinam princípios de disponibilidade dos equipamentos, confiabilidade, qualidade do produto, segurança e meio ambiente. Através desses princípios, a MCC busca desenvolver um programa ótimo de manutenção (BLOOM, 2006).

Segundo Rausand (1998), a MCC possibilita a redução de custos de manutenção por meio da priorização das funções mais importantes do 
sistema, agregando atividades de manutenção estritamente necessárias para a continuidade dessas funções e evitando ou removendo ações de manutenção desnecessárias.

A base de trabalho da MCC pode ser representada através de uma FMEA modificada, onde se definem as funções e padrões de desempenho dos equipamentos, seus modos de falha, as causas e consequências destas e, consequentemente, a criticidade da falha para a linha de produção e o tipo de manutenção recomendada - preventiva, corretiva, preditiva ou rodar até a falha (BLO0M, 2006; FOGLIATTO; RIBEIRO, 2008).

Um estudo mais cuidadoso da literatura revela que, embora muitos livros e artigos tratem da MCC, a grande maioria aborda apenas as questões qualitativas, deixando uma lacuna quanto às questões quantitativas, as quais poderiam efetivamente contribuir para o aumento da confiabilidade dos sistemas (RA0 et al., 1996).

Wilmeth e Usrey (2000) e Rao et al. (1996) afirmam que, apesar de a MCC ser um excelente método para a definição de listas de atividades de manutenção, ela usualmente não aborda questões relacionadas à definição da periodicidade dessas atividades. Rausand (1998) explica que a determinação de intervalos ótimos entre as manutenções é difícil porque deve ser baseada em informações sobre taxa de falhas e custos das falhas e da manutenção preventiva. Na mesma linha, Rao et al. (1996) relatam que abordagens qualitativas têm sido preferidas às abordagens quantitativas devido à falta de dados históricos das plantas e de métodos estatísticos adequados para interpretar esses dados. Complementando suas ideias, Rausand (1998) afirma que os artigos sobre otimização da manutenção são escritos por pesquisadores matemáticos ou estatísticos que utilizam uma linguagem incompreensível e propõem modelos de complexidade muito além do aceitável na prática da manutenção.

Assim, o objetivo principal deste artigo é analisar o tipo de suporte quantitativo que as empresas necessitam para a operacionalização da MCC. Partindo da hipótese de que tanto a literatura como a prática empresarial concentram-se em abordagens qualitativas, ele busca identificar o suporte quantitativo necessário à MCC. Para tanto, é elaborada uma revisão de literatura e, posteriormente, são realizadas entrevistas em empresas que executam manutenção. Como resultado final, tem-se a elaboração de um quadro comparativo e um modelo conceitual que associa os métodos quantitativos levantados às atividades da MCC.
Vale ressaltar que o estudo do suporte quantitativo necessário à operacionalização da MCC é importante para que, posteriormente, possam ser desenvolvidos procedimentos que incluam essa abordagem quantitativa na programação da manutenção. Esses procedimentos podem auxiliar as empresas a reduzir custos de manutenção e a aumentar a disponibilidade e confiabilidade de seus equipamentos.

Este artigo está estruturado em cinco seções. $\mathrm{Na}$ seção 2 é apresentado o referencial teórico sobre a utilização de métodos quantitativos na manutenção centrada em confiabilidade. Na seção 3 são mostrados os procedimentos metodológicos utilizados para realizar o trabalho e solucionar o problema de pesquisa. Os resultados obtidos, bem como sua discussão, estão na seção 4. Por fim, na seção 5, são apresentadas as conclusões referentes ao estudo realizado.

\section{Referencial teórico}

0 principal objetivo da MCC é criar uma rotina de manutenção estratégica que preserve funções de sistemas e equipamentos de forma efetiva e com custos aceitáveis (WILMETH; USREY, 2000). Se os custos de uma manutenção preventiva forem maiores que o custo associado às perdas operacionais e ao reparo, a manutenção não é vantajosa (RAO et al., 1996), a menos que se trate de um requisito normativo ou relacionado à segurança ou meio ambiente (BLO0M, 2006).

A base de trabalho de um programa de MCC é a definição das funções e padrões de desempenho dos equipamentos, seguida da descrição de suas possiveis falhas, bem como da análise de suas causas, consequências e da definição de ações que impeçam ou amenizem sua ocorrência (FOGLIATTO; RIBEIR0, 2008). Nesse contexto, adaptações da FMEA são ferramentas amplamente utilizadas na MCC. Rausand (1998) sugere a utilização da FMECA (failure modes, effects and criticality analysis) para a identificação de modos de falha e definição de sua criticidade. Bloom (2006) apresenta a COFA (consequence of failure analysis) como uma forma mais acurada e simples de definir as consequências das falhas. Fogliatto e Ribeiro (2008) recomendam a utilização de uma FMEA ampliada que contenha também informações pertinentes às atividades de manutenção.

Alguns elementos importantes para entender a MCC são as falhas escondidas e as falhas múltiplas. Falhas escondidas ocorrem quando a falha não é evidente para o operador, sendo que o sistema 
permanece em funcionamento até o surgimento de uma segunda falha em outro componente. Falhas múltiplas acontecem exatamente quando a ocorrência de uma falha está escondida, o que diminui a confiabilidade do sistema, e este pode perder sua função a qualquer momento, devido ao aparecimento de uma segunda falha. Esses tipos de falhas ocorrem em sistemas com redundância ou em sistemas que possuem backups normalmente inativos (BLO0M, 2006; RAUSAND, 1998).

A otimização da manutenção requer uma combinação balanceada entre os três tipos de manutenção: i) preventiva, podendo ser relacionada diretamente com a idade do componente ou procura por falhas escondidas; ii) preditiva, diretamente relacionada com a condição do componente, também conhecida como preventiva baseada em inspeções; e iii) corretiva, utilizada em falhas aleatórias e em itens determinados para rodar até a falha (FARRERO et al., 2002; DESHPANDE; MODAK, 2002).

Alguns autores recomendam a utilização de métodos qualitativos para a definição do tipo de manutenção e do intervalo entre manutenções a ser adotado. Deshpande e Modak (2002), Rausand (1998), Bloom (2006), Fogliatto e Ribeiro (2008) e Rao et al. (1996) apresentam diagramas de decisão semelhantes para a identificação do tipo de manutenção recomendada. Esses diagramas utilizam informações referentes à evidência da falha, suas consequências e aplicabilidade das atividades de manutenção. Bloom (2006) sugere a utilização da experiência da equipe de manutenção para estabelecer o intervalo entre manutenções, contudo destaca que revisões do plano de manutenção devem ser constantes e baseadas em feedbacks relativos às condições da atividade de manutenção.

Visando uma abordagem quantitativa, a definição do tipo de manutenção a ser adotada para cada componente depende da densidade de probabilidade característica das falhas deste componente. Através da análise de dados de falhas pode-se determinar qual o comportamento das falhas desse equipamento ao longo do tempo e, dessa forma, escolher o tipo de manutenção mais adequada (LAFRAIA, 2001; SELLITTO, 2005).

A probabilidade condicional de falhas no tempo depende do tipo de componente que está sendo analisado. Algumas dessas probabilidades, apresentadas na Figura 1, são: a) a curva da banheira, onde há uma elevada taxa de falha no momento em que o componente começa a operar, seguida de uma estabilização onde ocorrem falhas aleatórias e finalizada pelo aumento considerável das falhas devido à fadiga e ao desgaste; b) taxa

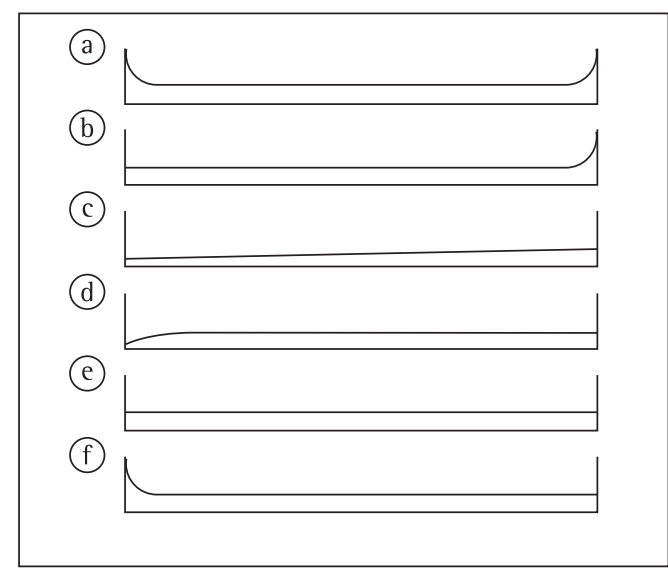

Figura 1. Tipos de probabilidades condicionais de falhas. Fonte: Moubray (1997).

de falhas constante, com crescimento acentuado no final do período devido a falhas relacionadas à idade, normalmente explica o comportamento de componentes mecânicos; c) taxa de falhas levemente crescente com o tempo; d) taxa de falha baixa no início da vida do componente, seguida de taxa de falha constante; e) taxa de falha constante durante toda a vida do componente, normalmente explica o comportamento de componentes eletrônicos; e f) taxa de falha elevada no início da vida do componente, com decréscimo acentuado e estabilização das falhas (LAFRAIA, 2001; SELLITTO, 2005).

Para Lafraia (2001), as distribuições dos tempos de falha dos componentes devem ditar a política de manutenção do sistema. Caso contrário, a manutenção pode ser ineficaz ou mesmo aumentar a taxa de falha do componente. Assim, a definição do tempo entre as manutenções depende de uma descrição matemática do processo de falha do componente (RAUSAND, 1998).

Sellitto (2005) sugere a utilização de um método quantitativo para definir tanto o tipo de manutenção quanto o intervalo entre manutenções. Após a coleta de tempos até falha e definição do modelo de distribuição que apresenta o melhor ajuste dos dados, determina-se se a máquina se encontra na fase de mortalidade infantil, maturidade ou desgaste. Para tanto, são utilizados testes estatísticos de processos aleatórios. Para equipamentos na mortalidade infantil, recomenda-se a manutenção corretiva; para aqueles que se encontram na fase de maturidade, podem ser adotadas estratégias de manutenção preditiva e corretiva; e, para equipamentos em fase de desgaste, sugere-se a utilização de manutenção preventiva e preditiva. 
Baseado em um estudo de caso, Sellitto (2005) também propõe a utilização dos tempos até falha das máquinas para o cálculo de três parâmetros de uma distribuição de Weibull. Assim, após definir o grau de significância, é possível calcular o fator de forma $\gamma$ e, consequentemente, determinar se a máquina possui taxa de falha decrescente $(\gamma<1=$ mortalidade infantil), taxa de falha constante $(\gamma=1=$ maturidade) ou taxa de falha crescente $(\gamma>1$ = desgaste). 0 intervalo de tempo entre manutenções preventivas é obtido considerando as relações de custos entre manutenções corretivas e preventivas.

Para determinar o tipo de manutenção mais apropriado e o intervalo adequado entre manutenções, Farrero et al. (2002) apresentam uma abordagem semelhante. Após coletar dados de tempos até falha, determina-se a distribuição de melhor ajuste para esses dados através da utilização dos métodos de papel de probabilidade e estimação de máxima probabilidade. Após estimar os parâmetros da distribuição, é possível determinar o tempo médio até falha, o tempo de vida médio e o tempo entre inspeções. 0 tipo de manutenção escolhido deve estar relacionado com o tipo de distribuição das falhas do sistema.

Shankar e Sahani (2003) propõem um complexo método numérico e estatístico que tem por objetivo determinar a quantidade total de manutenção necessária para um dado número de unidades funcionando no tempo. 0 método pressupõe o uso combinado de conhecimentos de probabilidade e seus modelos de distribuição, custos de manutenção e possibilidades de investimento na manutenção.

Um método quantitativo focado no menor custo anual de manutenção é proposto por Silva et al. (2008). Equações diferentes para cada estratégia de manutenção são propostas para o cálculo do custo anual de manutenção. Para tanto, é necessário saber, de antemão, o custo unitário de cada tipo de manutenção, a taxa de falha para cada tipo de manutenção, o tempo anual do equipamento em operação e o número anual de intervenções de manutenção. 0 estudo revela que o uso combinado de manutenções corretivas, preventivas e preditivas, além de reduzir o custo anual total da manutenção, aumenta os índices de disponibilidade e confiabilidade da planta.

Rao et al. (1996) sugerem um método para a definição da periodicidade de manutenção com ênfase na máxima disponibilidade dos equipamentos e um custo ótimo de manutenção. Assume-se que os tempos até falha coletados seguem uma distribuição de Weibull e estimam-se seus parâmetros. 0 tempo médio até falha é obtido através de um algoritmo que utiliza como entrada os parâmetros estimados da distribuição de Weibull. 0 intervalo ótimo entre manutenções e o custo de manutenção por unidade do tempo é definido através de outro algoritmo que utiliza como dados de entrada o custo da manutenção preventiva e das paradas e os parâmetros da distribuição de Weibull. Os algoritmos são resolvidos utilizando-se softwares de programação computacional.

Zhang e Nakamura (2005) propõem um método para otimizar a programação das manutenções baseado em uma lista dos elementos em ordem decrescente de efeito na manutenção. 0 efeito dos elementos na manutenção é quantitativamente definido como o aumento da disponibilidade do sistema causado pelo aumento das disponibilidades dos elementos vizinhos com seus intervalos de manutenção. A proposta central do método é o aumento do número de atividades de manutenção em alguns elementos que possuem um grande efeito na produção, a fim de aumentar a disponibilidade do sistema, juntamente com a diminuição do número de atividades de manutenção naqueles elementos que possuem um baixo efeito, com o propósito de reduzir o número total de atividades de manutenção.

Um método quantitativo para a otimização dos níveis de estoque de itens consumíveis derivado de um programa de MCC é proposto por Farrero et al. (2002). Esse método baseia-se no conhecimento da taxa de falha do componente $(\lambda)$, do nível de confiabilidade desejado e da escala de tempo que o estoque deve suprir. 0 estoque necessário é obtido através da multiplicação da taxa de falha pelo tempo de funcionamento do componente dentro da escala de tempo total que o estoque deve suprir $(H)$, seguido da identificação na tabela da distribuição de Poisson do valor $m$ correspondente ao nível de confiabilidade desejado e ao valor de $\lambda \times H$.

Lafraia (2001) apresenta um método para otimização da frequência de manutenção preventiva baseado na maximização da disponibilidade média e minimização do custo total de manutenção. Supondo que a manutenção preventiva seja perfeita, ou seja, reduz a taxa de falha ao valor inicial para taxas de falha $(\lambda)$ linearmente crescente, decrescente ou constante no tempo, pode-se calcular a disponibilidade média através da taxa de falha média:

$$
D m \cong \frac{1}{1+\frac{\lambda c m}{\mu c}+\frac{\lambda p}{\mu p}}
$$

0 problema é solucionado através da maximização da equação anterior com relação à $\lambda p$ onde: 


$$
\lambda c m=\frac{1}{1 / \lambda_{p}} \int_{0}^{1 / \lambda p} \lambda c(t) \cdot d t
$$

Onde $D m$ é a disponibilidade total, $\lambda$ cé a taxa de falha, $\mu$ cé a taxa de reparo de manutenção corretiva, $\lambda p$ é a frequência de manutenção preventiva, $\mu p$ é a taxa de ação de manutenção preventiva e $\lambda \mathrm{cm}$ é a taxa de falha média. Igualando a zero a primeira derivada da função $D m$ em relação a $\lambda p$ tem-se a frequência ótima de manutenção preventiva:

$$
\lambda p^{*}=\sqrt{\frac{\alpha r \mu p}{2 \mu c}}
$$

Onde $\alpha$ r é o aumento da taxa de falha/ano por ano ou o coeficiente da equação linear da taxa de falha. Para a minimização do custo de manutenção tem-se a seguinte equação para determinar a frequência de manutenção preventiva:

$$
\lambda p^{*}=\sqrt{\frac{\alpha r C r}{2 C p}}
$$

Onde $\mathrm{C} r$ é o custo médio de uma manutenção corretiva e $\mathrm{C} p$ é o custo médio de uma manutenção preventiva.

\section{Método de trabalho}

Quanto a sua natureza, esta pesquisa classifica-se como pesquisa aplicada, onde se busca gerar conhecimentos para aplicação prática dirigidos à solução de problemas específicos. Quanto à forma de abordagem do problema, esta classifica-se como pesquisa qualitativa. A base da pesquisa qualitativa é a interpretação dos fenômenos e a atribuição de significados, não requerendo o uso de métodos e técnicas estatísticas (SILVA; MENEZES, 2005).

Do ponto de vista de seus objetivos, esta pesquisa classifica-se como exploratória, pois visa proporcionar maior familiaridade com o problema visando torná-lo explícito. Envolve levantamento bibliográfico e entrevistas com pessoas que tiveram experiências práticas com o problema pesquisado. Quanto aos seus procedimentos técnicos, ela é do tipo estudo de campo, visto que a pesquisa é desenvolvida por meio da observação direta das atividades do grupo estudado e de entrevistas com informantes para captar suas explicações e interpretações do que ocorre na prática (GlL, 1991).

Este trabalho foi realizado nas seguintes etapas: i) identificar as atividades essenciais da manutenção centrada em confiabilidade (MCC); ii) identificar o suporte quantitativo que a MCC necessita; iii) realizar entrevistas em empresas que executam manutenção, a fim de verificar se utilizam os métodos quantitativos descritos na literatura e, paralelamente, identificar outros métodos quantitativos que sejam necessários, mas que não foram revelados pela literatura; e iv) elaborar um quadro comparativo associando métodos quantitativos e atividades da MCC.

Para identificar as atividades essenciais da MCC foi realizada uma revisão da literatura existente, gerando um quadro das atividades essenciais para a operacionalização da MCC. A identificação do suporte quantitativo que a MCC necessita foi obtida através da revisão de literatura e elaboração de quadro resumo dos métodos quantitativos publicados e resultados que eles oferecem.

$\mathrm{Na}$ etapa de realização de entrevistas em empresas que executam manutenção, para verificar se utilizam os métodos quantitativos e, paralelamente, identificar outros métodos que possam ser necessários e que não são reportados na literatura, foi empregada a entrevista não estruturada focalizada. 0 relato dessas entrevistas sumariza as percepções relativas ao MCC de profissionais que atuam na gestão da manutenção.

Para Marconi e Lakatos (2005), na entrevista não estruturada, o entrevistador tem liberdade para desenvolver cada situação em qualquer direção que considere adequada. Classifica-se como focalizada devido à existência de um roteiro de tópicos relativos ao problema, geralmente em forma de perguntas abertas, sendo que o entrevistador tem liberdade de formular as questões que quiser: sonda razões e motivos, esclarece situações, não obedecendo, a rigor, a uma estrutura formal.

0 quadro comparativo associando métodos quantitativos e atividades da MCC foi elaborado através da união dos resultados de atividades e métodos das etapas $i$ e $i i$. Esse quadro visa relacionar as principais atividades da MCC aos métodos quantitativos que podem fornecer suporte a essas atividades.

\section{Resultados e discussão}

Inicialmente, é preciso estabelecer o conjunto de atividades essenciais para a operacionalização da MCC. Bloom (2006) indica que essas atividades são: definição e treinamento das pessoas que trabalham na manutenção, listagem e classificação de criticidade dos componentes conforme suas funções, classificação e análise de falhas funcionais e classificação da abordagem de manutenção (preventiva, preditiva e corretiva).

Rausand (1998) e Rao et al. (1996) identificam como essenciais as seguintes atividades da MCC: classificação dos componentes segundo a classificação de suas funções, determinação 
dos seus padrões de desempenho, classificação e análise de falhas funcionais, classificação do tipo de manutenção a ser aplicado e definição e monitoramento de indicadores (taxa de falhas, tempo médio até falhas, tempo médio de reparo).

Lafraia (2001) e Sellitto (2005) relacionam à MCC atividades mais quantitativas: definição e monitoramento de indicadores (disponibilidade dos equipamentos $A(t)$, confiabilidade $R(t)$, custo da manutenção), análise quantitativa das falhas (risco ou taxa de falha $\mathrm{h}(\mathrm{t})$, distribuição de probabilidade das falhas) e programação de manutenção (definição de tarefas e intervalos). Lafraia (2001) e Silva et al. (2008) acrescentam o dimensionamento de estoque de peças de manutenção.

Fogliatto e Ribeiro (2008) destacam como questões básicas da MCC: definição e capacitação da equipe de manutenção, definição de funções e classificação dos componentes e de seus padrões de desempenho, análise de falhas, classificação do tipo de manutenção, seleção das atividades de manutenção e estabelecimento de indicadores.

Considerando a contribuição dos autores citados, o Quadro 1 apresenta um resumo das atividades que podem ser consideradas essenciais para a operacionalização da MCC.

A seguir, é preciso estabelecer os métodos quantitativos que podem facilitar e qualificar a operacionalização das atividades essenciais da MCC (listadas no Quadro 1). Para a análise das falhas é recomendada a utilização dos modelos de probabilidade condicional das falhas, a fim de determinar o comportamento das falhas no tempo, associados a estudos de confiabilidade para identificar o comportamento dos tempos até falha de cada componente (LAFRAIA, 2001; SELLITTO, 2005; FARRERO et al., 2002).
A classificação dos componentes depende da classificação de suas funções, da classificação de suas falhas e dos resultados da análise de falhas. Normalmente, pode ser resolvida de forma qualitativa com a utilização da FMEA, contudo, para sistemas mais complexos com vários níveis de redundância pode ser necessária a utilização de diagramas de blocos ou simulação de Monte Carlo (RAUSAND, 1998).

A classificação da abordagem de manutenção necessita do uso de distribuições de probabilidade aplicadas à confiabilidade (SELLITTO, 2005; FARRERO et al., 2002). Isso é necessário para análise prévia das falhas e identificação da distribuição probabilística que melhor ajuste os dados de tempos até falha. Através da determinação dos parâmetros das distribuições de probabilidade, é possível estabelecer se o componente está em fase de falhas prematuras, maturidade ou desgaste. 0 tipo de manutenção adequado depende da fase em que o componente se encontra.

A atividade de programação da manutenção pode ser dividida em duas outras atividades: determinação dos intervalos entre manutenções preventivas e programação da execução das atividades de manutenção. A determinação dos intervalos ótimos entre manutenções preventivas requer o uso de distribuições de probabilidade para a análise dos tempos de bom funcionamento dos componentes e equipamentos envolvidos. Os três métodos de cálculo de intervalo ótimo que foram identificados na literatura enfatizam, ainda que de formas diferentes, a máxima disponibilidade do equipamento a um custo ótimo (RAO et al., 1996; ZHANG; NAKAMURA, 2005; LAFRAIA, 2001).

Para a programação das atividades de manutenção é necessário o agrupamento de

Quadro 1. Atividades essenciais da MCC.

\begin{tabular}{|c|c|}
\hline Atividade & Escopo \\
\hline $\begin{array}{l}\text { 1. Definição e capacitação da equipe } \\
\text { de manutenção }\end{array}$ & $\begin{array}{l}\text { Contempla a escolha da equipe de manutenção, atribuição de funções e treinamento para que a } \\
\text { equipe possa cumprir suas funções }\end{array}$ \\
\hline 2. Listagem de componentes & $\begin{array}{l}\text { Envolve a identificação de todos os equipamentos do setor, devidamente desdobrados em conjuntos } \\
\text { e componentes }\end{array}$ \\
\hline 3. Classificação de funções & $\begin{array}{l}\text { Contempla a classificação da função de cada componente em função essencial ou função auxiliar. } \\
\text { Função essencial é o objetivo principal da instalação do componente e função auxiliar é aquela } \\
\text { requerida para dar suporte a uma função essencial }\end{array}$ \\
\hline 4. Classificação de falhas & Compreende a classificação das falhas em falhas evidentes ou falhas escondidas \\
\hline 5. Análise de falhas & Envolve a análise do comportamento dos tempos até falha ao longo do tempo dos componentes \\
\hline 6. Classificação de componentes & $\begin{array}{l}\text { Contempla a classificação dos componentes quanto às falhas funcionais em: componentes críticos, } \\
\text { potencialmente críticos, economicamente significativos e não críticos }\end{array}$ \\
\hline $\begin{array}{l}\text { 7. Classificação da abordagem de } \\
\text { manutenção }\end{array}$ & $\begin{array}{l}\text { Contempla a escolha da abordagem de manutenção mais apropriada (corretiva, preventiva ou } \\
\text { preditiva) }\end{array}$ \\
\hline 8. Programação da manutenção & $\begin{array}{l}\text { Envolve o agrupamento de atividades menores em pacotes de manutenção e o planejamento de sua } \\
\text { execução em termos de tempo e sequenciamento }\end{array}$ \\
\hline 9. Dimensionamento de estoques & $\begin{array}{l}\text { Compreende a determinação do tamanho do estoque de cada componente necessário para suprir } \\
\text { determinado nível de serviço }\end{array}$ \\
\hline 10. Monitoramento de indicadores & $\begin{array}{l}\text { Contempla a definição e monitoramento de indicadores do processo de manutenção de } \\
\text { equipamentos }\end{array}$ \\
\hline
\end{tabular}


atividades menores em pacotes de manutenção que devem ser executados ao mesmo tempo ou numa sequência específica (RAUSAND, 1998). Sistemas de MRPIl podem ser utilizados para programar os pacotes de atividades, considerando-se que as datas de entrega são os prazos de execução de cada pacote de manutenção e que cada pacote é independente e não possui precedências. Para o sequenciamento das atividades dentro de um pacote de manutenção em uma máquina, pode-se utilizar o método mean flow time (MFT), conforme descrito em Elsayed e Boucher (1994).

0 dimensionamento dos estoques de componentes de manutenção pode ser obtido através da utilização de distribuições de probabilidade, juntamente com modelos de gestão de estoques estocásticos ou determinísticos. As distribuições de probabilidade servem para avaliar o comportamento dos tempos até a falha dos componentes e determinar as demandas de peças de reposição e materiais de consumo. Os modelos de gestão de estoques, por sua vez, podem ser utilizados para determinar o ponto de reposição ou o intervalo de revisão do estoque. Modelos estocásticos que apresentam demanda e lead time como variáveis aleatórias independentes no período de análise costumam ser mais eficientes do que modelos determinísticos, porém a escolha do modelo depende das características da organização e dos dados disponíveis. Um método simplificado para otimização de níveis de estoques de itens consumíveis foi apresentado na literatura por Farrero et al. (2002).

0 monitoramento de indicadores é essencial para medir e verificar o atual desempenho do sistema de manutenção. Geralmente são apresentados na literatura os indicadores de confiabilidade tempo médio entre falhas (MTBF), tempo médio entre reparos (MTTR) e disponibilidade (A). Uma alternativa de medida global do sistema é o indicador de tempo médio entre interrupções (MTBI), que considera todos os minutos de interrupção e não apenas o tempo de falha do equipamento (HANSEN, 2006). Também é possível utilizar indicadores de eficiência global (OEE) e de produtividade efetiva total (TEEP) dos equipamentos. A OEE mede a eficácia do processo em fazer bons produtos na velocidade considerada e no tempo que o equipamento está programado para operar, enquanto a TEEP mede o percentual do tempo de calendário total que o equipamento opera a uma velocidade ideal, produzindo bons produtos.

A maioria dos autores que discorre sobre a MCC enfatiza a importância do controle de custos (SILVA et al., 2008; HAUGE, 2002; RAO et al., 1996; WESSELS, 2003; WILMETH; USREY, 2000; ZHANG; NAKAMURA, 2005; LAFRAIA, 2001).
Assim, é evidente que sistemas de custeio, como o ABC (Activity Based Costing) ou outros, devem ser incluídos entre as técnicas quantitativas que fornecem suporte à operacionalização da MCC.

Complementarmente, para verificar se os métodos quantitativos descritos na literatura eram utilizados na prática pelas equipes de manutenção das empresas, bem como para identificar outros métodos quantitativos que possam ser necessários, foram realizadas entrevistas focalizadas não estruturadas com os responsáveis pela manutenção em sete empresas dos setores metal-mecânico e eletrônico. Todas são empresas de grande porte, que se destacam em seus mercados específicos. Os principais produtos dessas empresas são: empresa 1, refrigeradores de ar; empresa 2, eixos cardans e chassis; empresa 3, estruturas metálicas; empresa 4, acessórios para automóveis; empresa 5, acessórios para caminhões; empresa 6, componentes elétricos; empresa 7, aços especiais.

Duas das sete empresas entrevistadas desconhecem os conceitos de MCC, as demais, apesar de conhecer, comentaram que não aplicam esses conceitos devido à alta complexidade e grande demanda de tempo e pessoal necessários. Normalmente, as empresas utilizam ferramentas como a FMEA e a análise de confiabilidade nas atividades do setor de engenharia de produtos, contudo esses conceitos não são transmitidos ao setor de manutenção, que desconhece as vantagens da aplicação dessas ferramentas na gestão da manutenção. Por outro lado, os conceitos de manutenção produtiva total (MPT) são amplamente difundidos nas empresas, sendo que todas elas afirmaram conhecer e aplicar na prática algum desses conceitos, destacando-se a manutenção autônoma.

As entrevistas revelaram que seis das sete empresas estudadas não utilizam métodos quantitativos direcionados à MCC. De modo geral, a explicação para a não utilização de tais métodos envolveu a percepção que eles demandariam muito tempo (e, portanto, muito custo) para sua operacionalização. Ainda que possuam históricos de falhas de equipamentos, as empresas se limitam a utilizar apenas métodos qualitativos para a gestão da manutenção, como os conceitos de gestão contemplados na manutenção produtiva total (MPT). A elaboração de planos de manutenção normalmente é feita através da utilização dos manuais dos equipamentos associada à experiência dos profissionais da manutenção.

Verificou-se que métodos quantitativos são utilizados somente para o monitoramento de indicadores como: custos da manutenção, disponibilidade, MTBF e MTTR por equipamento. Porém, é importante ressaltar que em três das 
sete empresas entrevistadas não existia nenhum indicador definido para monitorar o processo de manutenção. 0 Quadro 2 apresenta os indicadores utilizados em cada empresa pesquisada.

Observou-se que, quanto a sua rotina de manutenção, as empresas estudadas podem ser divididas em dois grupos: as que utilizam e as que não utilizam indicadores no processo de manutenção.

Em geral, aquelas que utilizam indicadores possuem um plano de manutenções preventivas preestabelecido para cada equipamento, sendo que ele é cumprido de acordo com a disponibilidade da produção para parar a máquina e a disponibilidade de pessoal de manutenção para executar a atividade. Assim, em empresas onde há pouca disponibilidade para parar o equipamento, muitas ocorrências de manutenção corretiva ou pouca mão de obra de manutenção, as tarefas previstas no plano de manutenção preventiva vão sendo adiadas e acumuladas, formando as chamadas backorders. Nessas empresas, os operadores podem solicitar manutenções corretivas e a prioridade das atividades depende da criticidade da falha em termos de segurança e parada de produção. Para aqueles equipamentos críticos (gargalos, com alto MTTR ou baixo MTBF, críticos com relação à segurança operacional), as falhas são analisadas a fim de determinar e bloquear sua causa.

As empresas que não utilizam indicadores apresentam um nível de desenvolvimento de gestão do processo de manutenção inferior às demais, limitando-se a estabelecer planos de manutenção preventiva que raramente são cumpridos e a executar manutenções corretivas.

No que se refere aos estoques de peças de reposição para a manutenção, constatou-se que, nos casos em que as empresas têm controle formal, o estoque mínimo ou ponto de reposição é definido com base nos históricos das falhas e na experiência, de forma intuitiva, sem a utilização de métodos específicos de gestão de estoques.

Uma das empresas entrevistadas destaca-se das demais, visto que emprega métodos quantitativos na gestão da manutenção. Nesta empresa, além dos métodos para monitoramento de indicadores

Quadro 2. Indicadores utilizados pelas empresas pesquisadas.

\begin{tabular}{|l|c|c|c|c|c|c|c|}
\hline \multirow{2}{*}{ Indicadores utilizados } & \multicolumn{7}{|c|}{ Empresas pesquisadas } \\
\cline { 2 - 8 } & 1 & 2 & 3 & 4 & 5 & 6 & 7 \\
\hline Custos da manutenção & $\bullet$ & $\bullet$ & $\bullet$ & - & - & - & $\bullet$ \\
\hline Disponibilidade $(A)$ & $\bullet$ & $\bullet$ & $\bullet$ & - & - & - & $\bullet$ \\
\hline MTBF & $\bullet$ & $\bullet$ & $\bullet$ & - & - & - & $\bullet$ \\
\hline MTTR & $\bullet$ & $\bullet$ & $\bullet$ & - & - & - & $\bullet$ \\
\hline Confiabilidade $(R(t))$ & - & - & $\bullet$ & - & - & - & - \\
\hline
\end{tabular}

comentados anteriormente, são utilizadas distribuições de probabilidade para determinar o comportamento das falhas dos equipamentos e identificar a necessidade de ações corretivas nos componentes de menor confiabilidade. Também é utilizado o método de simulação de Monte Carlo visando estimar a capacidade da linha de produção submetida a manutenções corretivas e programadas, bem como para prever a quantidade de manutenções necessárias para o próximo período. Através das tradicionais fórmulas de confiabilidade, são determinadas: a taxa de falhas $h(t)$, a distribuição de probabilidade das falhas $f(t)$ e a confiabilidade $R(t)$ por equipamento e, para os equipamentos críticos, por componente. A operacionalização desses métodos é suportada pela utilização de softwares computacionais estatísticos e de confiabilidade. 0 responsável pela manutenção ainda destacou o custo do ciclo de vida (LCC) como uma técnica da engenharia econômica importante para a determinação e análise dos custos que compõem o custo total de operacionalização do equipamento.

No Quadro 3, são apresentados os resultados da associação das principais atividades da MCC com os métodos quantitativos descritos na literatura e os métodos quantitativos citados por profissionais. Também é feita uma breve descrição sobre as contribuições de cada método para a MCC.

$\mathrm{Na}$ Figura 2 são relacionadas de forma simplificada as atividades essenciais da MCC com os métodos quantitativos observados na literatura e na prática empresarial. Observa-se que as "distribuições de probabilidade" têm papel fundamental para a MCC, visto que são usadas como suporte em várias de suas principais atividades. Os demais métodos são utilizados de forma mais localizada, junto a uma ou duas atividades.

Os métodos de engenharia econômica e a simulação de Monte Carlo também merecem destaque, pois cada um deles suporta duas atividades consideradas essenciais no âmbito da MCC. Devido às características desses métodos, eles permitem análises mais sofisticadas associadas ao custo e desempenho de sistemas produtivos sujeitos a manutenção.

Os demais métodos quantitativos, apesar de suportarem diretamente apenas uma atividade essencial, também dão contribuição expressiva. 0 uso de diagrama de blocos e técnicas de análise da confiabilidade de sistemas permite uma visão mais completa dos equipamentos e seus componentes críticos. Os métodos de programação de atividades, MRPII/MFT (mean flow time) ou similares, qualificam a alocação de recursos às atividades de manutenção, reduzindo custos e aumentando a produtividade. Os modelos estocásticos e 
Quadro 3. Quadro comparativo das atividades da MCC e métodos quantitativos.

\begin{tabular}{|c|c|c|c|}
\hline Atividades da MCC & $\begin{array}{c}\text { Métodos } \\
\text { quantitativos citados } \\
\text { na literatura }\end{array}$ & $\begin{array}{l}\text { Métodos } \\
\text { quantitativos citados } \\
\text { por profissionais }\end{array}$ & Contribuições dos métodos \\
\hline $\begin{array}{l}\text { Definição e capacitação da equipe de } \\
\text { manutenção }\end{array}$ & - & - & - \\
\hline Listagem de componentes & - & - & - \\
\hline Classificação de funções & - & - & - \\
\hline Classificação de falhas & - & - & - \\
\hline Análise de falhas & $\begin{array}{l}\text { Distribuições de } \\
\text { probabilidade }\end{array}$ & $\begin{array}{l}\text { Distribuições de } \\
\text { probabilidade }\end{array}$ & $\begin{array}{l}\text { Avaliar a distribuição dos tempos até a falha dos } \\
\text { componentes }\end{array}$ \\
\hline \multirow[t]{2}{*}{ Classificação de componentes } & $\begin{array}{c}\text { Diagrama de blocos } \\
\text { e técnicas de análise } \\
\text { da confiabilidade de } \\
\text { sistemas }\end{array}$ & - & $\begin{array}{l}\text { Identificar componentes críticos } \\
\text { Identificar redundâncias }\end{array}$ \\
\hline & $\begin{array}{l}\text { Simulação de Monte } \\
\text { Carlo }\end{array}$ & - & Identificar componentes críticos \\
\hline \multirow{2}{*}{$\begin{array}{l}\text { Classificação da abordagem de } \\
\text { manutenção }\end{array}$} & $\begin{array}{l}\text { Distribuições de } \\
\text { probabilidade }\end{array}$ & $\begin{array}{l}\text { Distribuições de } \\
\text { probabilidade }\end{array}$ & $\begin{array}{l}\text { Determinar a fase de vida do componente } \\
\text { (inicial, maturidade, desgaste) para determinar a } \\
\text { abordagem de manutenção mais apropriada }\end{array}$ \\
\hline & - & $\begin{array}{l}\text { Métodos de } \\
\text { Engenharia } \\
\text { Econômica }\end{array}$ & $\begin{array}{l}\text { Determinar o custo do ciclo de vida do } \\
\text { equipamento, conforme a abordagem de } \\
\text { manutenção escolhida }\end{array}$ \\
\hline \multirow{4}{*}{ Programação da manutenção } & $\begin{array}{l}\text { Distribuições de } \\
\text { probabilidade }\end{array}$ & $\begin{array}{l}\text { Distribuições de } \\
\text { probabilidade }\end{array}$ & $\begin{array}{l}\text { Estimativa de tempos de falha, tempos de reparo } \\
\text { e disponibilidade, para definição de intervalos } \\
\text { ótimos de manutenção }\end{array}$ \\
\hline & - & $\begin{array}{l}\text { Simulação de Monte } \\
\text { Carlo }\end{array}$ & $\begin{array}{l}\text { Estimativa da capacidade da linha de produção } \\
\text { submetida a manutenções corretivas e } \\
\text { programadas }\end{array}$ \\
\hline & - & $\begin{array}{l}\text { Métodos de } \\
\text { engenharia } \\
\text { econômica }\end{array}$ & $\begin{array}{l}\text { Identificar a influência do intervalo de } \\
\text { manutenção no custo do ciclo de vida do } \\
\text { equipamento } \\
\text { Identificar o momento de substituição do } \\
\text { equipamento }\end{array}$ \\
\hline & $\begin{array}{l}\text { MRPIl } \\
\text { MFT (mean flow } \\
\text { time) }\end{array}$ & - & $\begin{array}{c}\text { Programar os pacotes de atividades no tempo e } \\
\text { sequenciar as atividades dentro de um pacote de } \\
\text { manutenção em uma máquina }\end{array}$ \\
\hline \multirow{2}{*}{ Dimensionamento de estoques } & $\begin{array}{l}\text { Distribuições de } \\
\text { probabilidade }\end{array}$ & - & $\begin{array}{c}\text { Avaliar a distribuição dos tempos até a falha dos } \\
\text { componentes e determinar as demandas de peças } \\
\text { de reposição e materiais de consumo }\end{array}$ \\
\hline & \begin{tabular}{|c|} 
Modelos estocásticos \\
e determinísticos de \\
gestão de estoques
\end{tabular} & $\begin{array}{l}\text { Modelos estocásticos } \\
\text { e determinísticos de } \\
\text { gestão de estoques }\end{array}$ & $\begin{array}{l}\text { Determinar o ponto de reposição ou o intervalo de } \\
\text { revisão do estoque }\end{array}$ \\
\hline \multirow{3}{*}{ Monitoramento de indicadores } & $\begin{array}{l}\text { Distribuições de } \\
\text { probabilidade }\end{array}$ & $\begin{array}{l}\text { Distribuições de } \\
\text { probabilidade }\end{array}$ & $\begin{array}{l}\text { Monitorar a distribuição do tempo entre falhas, } \\
\text { do tempo para reparo e da disponibilidade dos } \\
\text { equipamentos para a tomada de ações }\end{array}$ \\
\hline & OEE, TEEP & - & $\begin{array}{c}\text { Medir a eficácia e produtividade do processo } \\
\text { produtivo }\end{array}$ \\
\hline & Sistemas de custeio & Sistemas de custeio & $\begin{array}{l}\text { Monitorar o custo da estratégia de manutenção } \\
\text { adotada pela empresa }\end{array}$ \\
\hline
\end{tabular}

determinísticos de gestão de estoques asseguram o devido dimensionamento das peças de reposição e materiais de consumo, minimizando a probabilidade de falta desses itens. 0 uso de indicadores específicos da manutenção, como OEE e TEEP, permite o acompanhamento das variáveis essenciais à produção, como disponibilidade, velocidade e qualidade. Os sistemas de custeio, como o sistema $\mathrm{ABC}$, permitem quantificar com precisão os custos da manutenção e seu impacto sobre os custos globais, revelando que a manutenção, devidamente planejada e dimensionada, reduz custos globais e aumenta a competitividade da empresa.

\section{Conclusão}

0 objetivo principal deste trabalho foi analisar o tipo de suporte quantitativo que as empresas necessitam para a operacionalização da MCC. Este estudo é importante para que, posteriormente, possam ser desenvolvidos procedimentos que incluam uma abordagem quantitativa na programação da manutenção. Esses procedimentos podem auxiliar as empresas a reduzir custos de manutenção e a aumentar a disponibilidade e confiabilidade de seus equipamentos. 


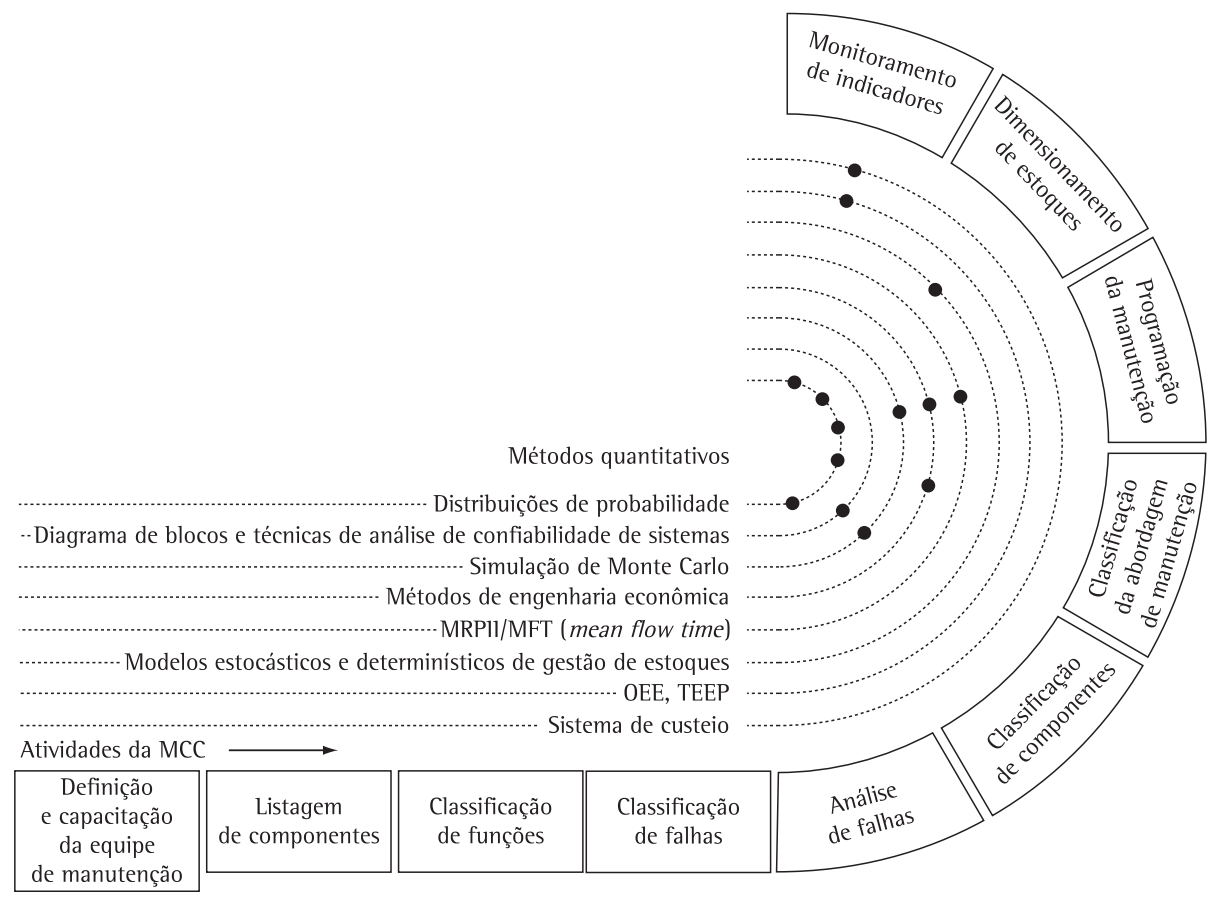

Figura 2. Atividades da MCC e métodos quantitativos.

Após a revisão da literatura e identificação das principais atividades da MCC, foram realizadas entrevistas com os responsáveis pela manutenção em sete empresas manufatureiras, a fim de verificar a utilização dos métodos encontrados na literatura e identificar outros métodos. 0 estudo da literatura indicou que há dez atividades essenciais para a operacionalização da MCC: definição e capacitação da equipe de manutenção, listagem de componentes, classificação de funções, classificação de falhas, análise de falhas, classificação de componentes, classificação da abordagem de manutenção, programação da manutenção, dimensionamento de estoques e monitoramento de indicadores.

0 estudo da literatura, reforçado por entrevistas junto a profissionais de empresas do setor metalmecânico, revelou que há oito métodos quantitativos importantes para sustentar a MCC. São eles: distribuições de probabilidade, diagrama de blocos e técnicas de análise de confiabilidade de sistemas, simulação de Monte Carlo, métodos de engenharia econômica, MRPII/MFT, modelos estocásticos e determinísticos de gestão de estoques, OEE/TEEP e sistemas de custeio.

A modelagem através de distribuições de probabilidade é crucial em muitas atividades da MCC, que não deve ater-se a valores médios, mas entender como tempos até a falha e tempos de reparo se distribuem. Os métodos de engenharia econômica qualificam a análise dos custos do ciclo de vida de máquinas e equipamentos. A simulação de Monte Carlo permite a análise de sistemas reais, de natureza essencialmente estocástica. 0 uso de diagrama de blocos e técnicas de análise da confiabilidade de sistemas permite uma visão mais completa dos equipamentos e de seus componentes críticos. Os métodos de planejamento e controle da produção, como o MRPIl, suportam o planejamento ótimo das atividades de manutenção. Os modelos estocásticos e determinísticos de gestão de estoques possibilitam resolver de forma ótima o conflito entre custos de armazenagem e custos de falta de peças de reposição. 0 uso de indicadores específicos da manutenção, como OEE e TEEP, permite o acompanhamento das variáveis essenciais em linhas de produção e o cálculo da eficiência global. Os sistemas de custeio, como o $\mathrm{ABC}$, possibilitam quantificar os custos do sistema produtivo, que incluem as atividades de manutenção.

A análise da contribuição desses métodos revela que eles têm potencial para fornecer uma contribuição expressiva à operacionalização da MCC. Em conjunto, o uso dos métodos quantitativos listados qualifica o planejamento, melhora 0 controle das atividades e reduz custos.

Por fim, vale ressaltar as delimitações deste estudo. Este trabalho limitou-se a identificar os métodos quantitativos que podem dar suporte às atividades da MCC. Aprofundamentos relativos à implementação e operacionalização desses métodos no processo de manutenção são sugestões para trabalhos futuros. Além disso, o estudo restringiu-se 
às sete empresas estudadas, pertencentes aos setores eletrônico e metal-mecânico. Não foram visitadas empresas de setores de maior risco, como por exemplo os setores aeroespacial, nuclear e petroquímico, que, sabidamente, apresentam maior desenvolvimento quanto a aplicações de manutenção e confiabilidade.

\section{Referências}

Bl00M, N. B. Reliability Centered Maintenance: implementation made simple. New York: McGraw-Hill, 2006.

DESHPANDE, V. S.; MODAK, J. P. Application of RCM to a medium scale industry. Reliability Engineering \& System Safety, v. 77, n. 1, p. 31-43, 2002.

ELSAYED, E. A.; BOUCHER, T. 0. Analysis and control of production systems. 2. ed. New Jersey: Prentice Hall, 1994.

FARRERO, J. C.; TARRÉS, L. G.; LOSILLA, C. B. Optimization of replacement stocks using a maintenance programme derived from reliability studies of production systems. Industrial Management \&t Data Systems, v. 102, n. 4, p. 188-196, 2002.

FOGLIATTO, F. S.; RIBEIRO, J. L. D. Confiabilidade e manutenção industrial. São Paulo: Campus - Elsevier, 2009.

Gil, A. C. Como elaborar projetos de pesquisa. 3. ed. São Paulo: Atlas, 1991.

HANSEN, R. C. Eficiência global dos equipamentos: uma poderosa ferramenta de produção/manutenção para aumento dos lucros. Porto Alegre: Bookman, 2006.

HAUGE, H. S. Optimizing intervals for inspection and failurefinding tasks. In: RELIABILITY AND MAINTAINABILITY SYMPOSIUM, 16., 2002. Seattle. Proceedings...

LAFRAIA, J. R. B. Manual de confiabilidade, mantenabilidade e disponibilidade. Rio de Janeiro: Qualitymark, 2001.

MARCONI, M. A; LAKATOS, E. M. Fundamentos de metodologia científica. 6. ed. São Paulo: Atlas, 2005.

MOUBRAY, J. Reliability-centered maintenance. 2. ed. New York: Industrial Press, 1997.
RAO, P. N.; SRIKRISHNA, S.; YADAVA, G. S. Reliabilitycentred maintenance applied to power plant auxiliaries. Journal of Quality in Maintenance Engineering, v. 2, n. 1, p. 3-14, 1996.

RAUSAND, M. Reliability centered maintenance. Reliability Engineering and System Safety, v. 60, n. 2, p. 121-132, 1998.

SELLITTO, M. A. Formulação estratégica da manutenção industrial com base na confiabilidade dos equipamentos. Revista Produção, v. 15, n. 1, p. 44-59, 2005.

SHANKAR, G.; SAHANI, V. Reliability analysis of a maintenance network with repair and preventive maintenance. International Journal of Quality $\&$ Reliability Management, v. 20, n. 2, p. 268-280, 2003.

SILVA, C. M.; CABRITA, C. M.; MATIAS, J. C. Proactive reliability maintenance: a case study concerning maintenance service costs. Journal of Quality in Maintenance Engineering. v. 14, n. 4, p. 343-355, 2008.

SILVA, E. L.; MENEZES, E. M. Metodologia da pesquisa e elaboração de dissertação. 4. ed. Florianópolis: Ed. UFSC, 2005.

WESSELS, W. R. Cost-Optimized scheduled maintenance interval for reliability-centered maintenance. In: RELIABILITY AND MAINTAINABILITY SYMPOSIUM, 17., 2003, Tampa. Proceedings...

WILMETH, G. W.; USREY, M. W. Reliability centered maintenance: a case study. Engineering Management Journal, v. 12, n. 4, p. 25-31, 2000.

ZHANG, T.; NAKAMURA, M. Reliability-based optimal maintenance scheduling by considering maintenance effect to reduce cost. Quality and Reliability Engineering International, v. 21, n. 2, p. 203-220, 2005.

\section{Agradecimentos}

Agradecemosà Coordenação de Aperfeiçoamento de Pessoal de Nível Superior (CAPES) e ao Conselho Nacional de Pesquisa e Desenvolvimento (CNPq) pelo fornecimento de bolsa de pesquisa. Também agradecemos às empresas entrevistadas, que colaboraram com a disponibilização de informações para este estudo.

\title{
The quantitative support necessary for the operation of Reliability Centered Maintenance (RCM)
}

\begin{abstract}
This article investigates the quantitative support necessary for the operation of Reliability Centered Maintenance (RCM). Initially, ten essential activities for the operation of RCM and quantitative methods that can support such activities were identified in the literature. Next, interviews were conducted in manufacturing companies to verify the use of the methods described in the literature and identify other methods used. As a result, a framework that associates the RCM essential activities with the quantitative methods was created, along with a brief description of each method contribution. Among the quantitative methods identified, probability distributions stood out for supporting various RCM activities. The Economic Engineering and Monte Carlo simulation methods were also emphasized since they enable a more sophisticated analysis related to cost and performance of production systems subjected to maintenance.
\end{abstract}

\section{Keywords}

Reliability centered maintenance. Quantitative methods. Metallurgical industry. Electronics industry. 